\title{
Making Components with Controlled Metal Deposition
}

\author{
Dr. Fernando Ribeiro \\ Industrial Electronics Dep. \\ University of Minho \\ 4800 Guimarães \\ Portugal
}

\author{
Prof. John Norrish \\ Faculty of Engineering \\ University of Wollongong \\ Northfields Avenue, Wollongong NSW 2522 \\ Australia
}

\begin{abstract}
Rapid Prototyping is a recent CAD/CAM/CIM based manufacturing technique which produces prototypes of components in a fraction of the time. This technique works by first drawing the part as a 3 Dimensional solid model using a CAD program and then 'printing' it in 3 Dimensions. The raw material can be a photopolymer or thermoplastic which solidifies when in contact with light. Other materials are available although $100 \%$ metal is not a very usual one.
\end{abstract}

This paper presents a new approach for a Rapid Prototyping technique which uses Robot Fusion Welding. The component is drawn as a 3D solid in a CAD package, and this is then electronically sliced in order to automatically generate the robot path. The welding parameters are also generated according to the desired material/thickness of the component. By serial link RS232-C, the robot program is then downloaded. This process works unmanned and proved to be good enough for the purpose.

This technique can be used to make not only the prototype but also the final component with the desired metal. Therefore, this represents also a new production technique more suitable for low volume production.

Another advantage with this new technique is that different metals can be used during the build up of the component to achieve different structural characteristics in different parts of the same component. This would not be possible with casting. The welding can be stopped at any time, the filler material changed, and the welding started again. The wire can be changed quickly.

The process created is hereby described as well as its advantages and disadvantages. Some case studies are carried out and described. Several other case studies were carried out and published in order to fully test this system and some references are made to those publications in section IV.

\section{INTRODUCTION}

The production planning of a new component can take, sometimes, months and this can mean a big loss of money since the markets are quickly and continuously changing. In order to make a company more competitive and able to respond to these changes in the market as quickly as possible, it would be very desirable to reduce this product-development cycle time. The most critical part of the whole process consists on the development of the prototype and therefore there is a need to reduce its manufacturing time. Several companies created a new concept on making prototypes and called it "Rapid Prototyping” processes.

The basis of a Rapid Prototyping technique consists on designing the component in a CAD system as a 3 Dimensions solid, and then an algorithm electronically slices that solid and gives instructions to a manufacturing system (CIM) which uses an additive process to add material and build the component from scratch. Tests can then be done to the prototype and should it not meet the requirements it can be changed in the CAD system and re-built again. The design engineer would often pefer to see a physical model of the component in hours instead of weeks. As a bottom line, these systems consist on a fast 3D printer.

\section{SYSTEM CONCEPT}

This system uses a similar principle as any other Rapid Prototyping system and basically it uses a welding robot to deposit welding beads in order to build up the component. This way metallic components are achieved and even different metals 
can be used on the same component unlike traditional casting techniques being only necessary to change the wire spool.

First a 3 Dimensional model of the shape is created using a CAD program (which in this case is AutoCAD). Depending on the complexity of the desired component, sometimes it is not possible to build the whole component in one go. Being so, the designer has to draw it in separate parts (separate solids) although close together. Each solid will be built separately and independently one after the other.

Information relative to each part of the component has to be 'input' into the system like the name of the part, width of the part, sequential order of build up for all the parts, orientation of the torch, etc. Some of this information will then be used to generate the appropriate welding parameters. After all these INPUT tasks are complete the next step is to slice each part. This is done automatically, being only necessary to select the proper option to start it in the software. This slicing procedure will generate polylines, in the CAD model, consisting on lines/arcs which represent the path that the robot has to follow (welding trajectory) to build up the desired part.

Once these polylines are created within the CAD model, the software is then ready to generate four outputs. The first one is a DXF file format which contains only the polylines (arcs/lines) which represent the path of the robot. The second output is the robot program itself for each part. These robot program consists of a text file with instructions in ARLA robot language (although it could be any other robot language). This robot program includes instructions to move the robot in such a way to follow the polylines and containing the start/stop welding instructions which were previously read from the 'Welding/Table Instructions' box. This task is represented by the 'Robot Program' box. The other two outputs conist on reports which contain instructions relating to the component itself: one is for the welding technician and contains instructions for each part of the component (welding parameters, timings, etc.) and the other is for the production manager and contains information about the component as a whole describing the information for each part (like the time it will take to be built, the quantity of material needed, etc.).

Once the robot program is created, this has to be compiled and downloaded to the robot. This task can be done by linking the computer to the robot with a serial cable through the RS-232-C port of the computer. A special program is necessary to perform this task. This particular binary file format is unique to each robot manufacturer. The robot program should now have been transferred to the robot and it is ready to run. When the robot starts welding, the 'Workpiece' starts growing in the planned shape.

\section{ADVANTAGES / DISADVANTAGES}

The main advantages of rapid prototyping techniques are:

a) reducing the prototyping time production

b) the techniques are 'automatic' which means there is less need to have a skilled operative although an operator is needed from time to time to control the process

c) these processes are less wasteful since they normally operate as an additive process

d) using a CAD (Computer Aided Design) program it is very easy to implement changes in the component and it is then only necessary to "re-print" the component

e) it is possible to leave the machine working with little supervision around the clock.

These main disadvantages are:

a) to produce a very smooth surface

b) it is necessary to machine the prototype. This quality surface is sometimes controllable by the layer thickness

c) the size of the prototype is limited by the size of the machine

d) the machines normally used are very costly

e) it is not always possible to make the prototype in the required material for final component (most of these techniques use wax, photopolymers, thermoplastics (PVC, nylon, etc.) and ceramics or even paper for the initial model)

f) Techniques which prototype components in $100 \%$ metal are not common although some attempts have been made.

The reason for making a prototype, should not only be to visualise but also usable to test and assess it in its final function. Therefore, it is important to make the prototype with the same material used in the real and final component. Also, to overcome the actual Rapid Prototyping processes disadvantages, a 
technique for rapid prototyping components using Robotic Arc Welding has been investigated. For making the component, a robot holding a welding torch is used to 'draw' the desired shape and this has to be moved around in a very precise and sometimes repetitive way. It is important to note that low price was a target to achieve in this project.

Similarly to other Rapid Prototyping techniques, this one also uses a $\mathrm{CAD}$ package to draw the component as a 3D solid (AutoCAD, Autodesk, USA). After drawn, the solid is sliced by a routine specially devised for the purpose and this was made using AutoLisp (to be compatible with the CAD system used). Then, a robot program is automatically generated, as well as the welding parameters, according to the desired material/thickness of the component. A simulation program (WorkSpace from Robot Simulations Ltd, Newcastle, UK) can be used to check the feasibility of the robot program generated against collisions and other problems (the simulation is not compulsory and only allows optimisation of the program). By serial link RS-232-C, the robot program is then downloaded to the robot (in this work an $\mathrm{ABB}$ Irb2000 was used) and the start button is pressed. This process works unmanned and proved to be good enough for the purpose. The welding technique used is MIG (Metal Inert Gas) but others could be used.

An example of a component being built by welding can be seen in Fig. 1.

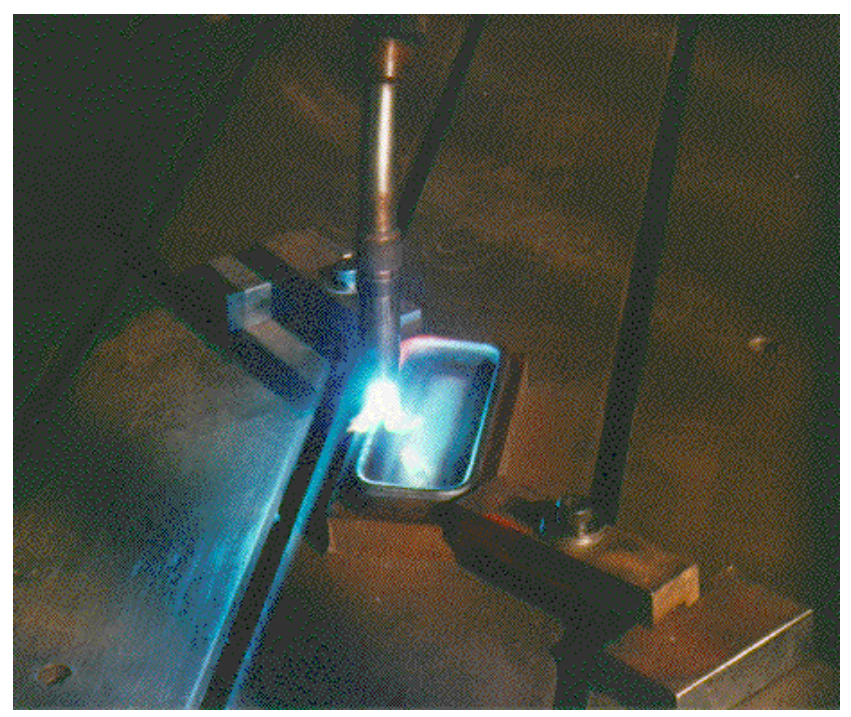

Fig. 1 - Rectangular component being made

\section{CASE STUDIES}

After the technique was developed, several tests were made in order to make sure that it would work. Several case studies with more complex shapes were created and checked against the expected shape. Also timings were carried out to check for time accuracy. Some of these case studies were presented at other conferences like the 'Portuguese Vase' [1], the 'square to round shape' [2], the 'pint glass' [3], a second 'square to round shape' [4]. The system itself and its concept has also been presented at [5]

\section{A. Small Vase}

This sample consists of a small vase shape around about 3 inches tall.

It took about 15 minutes to draw it in a CAD system, to slice, to generate the reports and the robot program. It took only about 15 minutes to make.

The width was planned to be $5 \mathrm{~mm}$ and in the end it was measured and it varied $+/-0.5 \mathrm{~mm}$. There was no mark in the vase where the robot moved up because it was done as a continuous spiral. The welding parameters are of extremely importance for the final dimensions, final surface finishing and final quality and these can make the whole difference between a good component and a bad component. The component here described can be seen in Fig. 2.

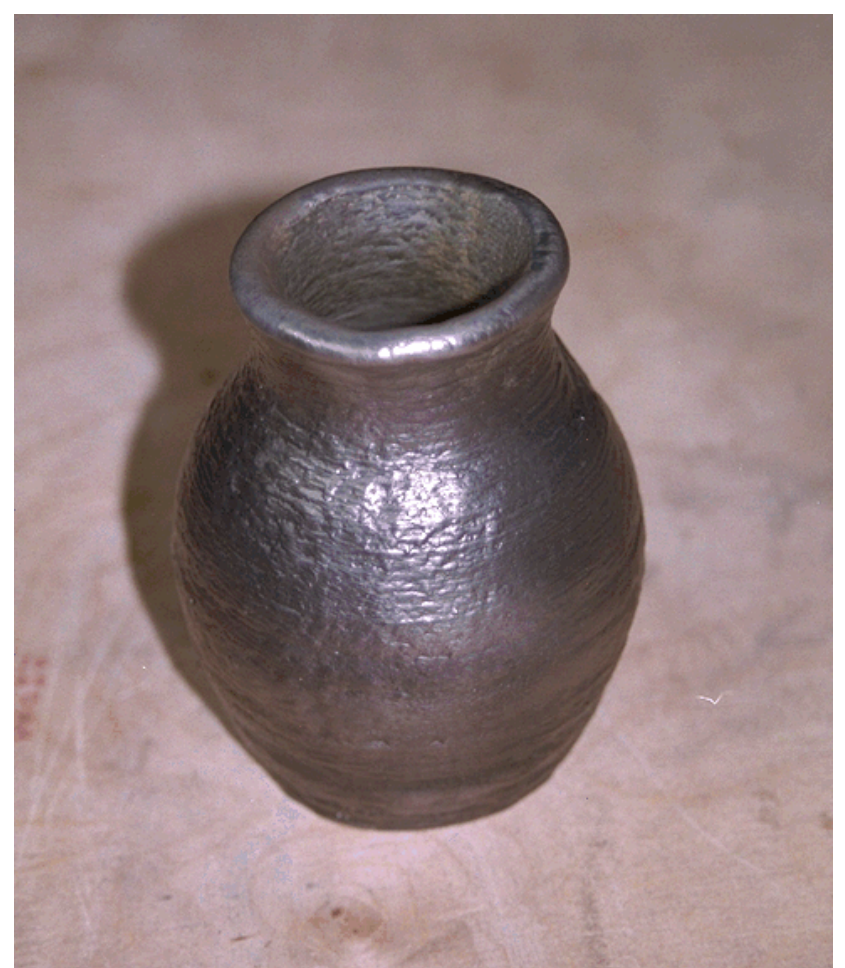




\section{B. Manifolds}

We were given a challenge to which we accepted straight away. The challenge consisted in making manifolds for a real car exhaust. Two different sizes were needed (two components each). These were drawn in about 15 minutes (both) and the slicing, generation of welding parameters, robot program, download to the robot, etc. took about another 15 minutes. The actual welding took around about 30 minutes each. These were welded to a base plate but this was then cut away as it can be seen in Fig. (two of them are already cut from the base). Basically, in one afternoon the four manifolds were made from scratch.

The car owner decided to machine them due to aesthetic reasons but just the outside. The inside was kept as it is because the tiny lines were used to reduce noise in the exhaust.

These larger ones are about $100 \mathrm{~mm}$ height and $50 \mathrm{~mm}$ radius and the others are about $100 \mathrm{~mm}$ height and $35 \mathrm{~mm}$ radius. The width was planned to be $5 \mathrm{~mm}$ and in the end it was measured and it varied +/- $0.2 \mathrm{~mm}$. This component was done as a spiral removing though a thicker location where the robot 'moves up'. Like in the previous component the welding parameters were of extremely importance for the final dimensions, final surface finishing and final quality and these can make the whole difference between a good component and a bad component. This component achieved a good surface finishing, very near geometrical expectations.

The components here described can be seen in Fig. 3.

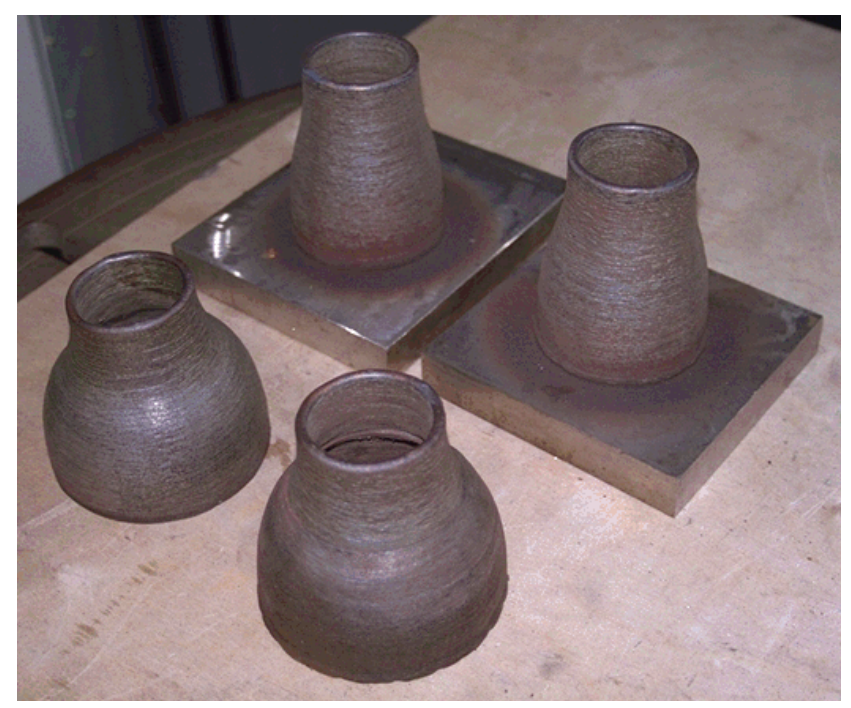

Fig. 3 - Four Manifolds actually being used at a car exhaust

\section{CONCLUSIONS}

1. This technique can be used to make not only the prototype but also the final component with the desired metal. Therefore, this represents also a new production technique more suitable for low volume production.

2. Another advantage with this new technique is that different metals can be used during the build up of the component to achieve different structural characteristics in different parts of the same component. This would not be possible with casting. The welding can be stopped at any time, the filler material changed, and the welding started again. The time to change the wire is not very long.

3. The technique here developed enables direct creation of metal objects from a CAD drawing.

4. To avoid incompatibility between software packages and the use of different computer platforms an attempt was successfully made to make all the software needed compatible with each other and working on the same platform. The minimum configuration of hardware and software was used. All the software was linked and as easy as possible to work with in order to reduce the learning time to a minimum.

5. After slicing the 3D shape, useful reports containing information relative to the welding parameters, quantity of consumables and welding tasks, were atomatically generated in order to improve the performance of the system, to control the process and to help the user to follow the progress of the whole process.

6. The robot program is automatically generated for most of the shapes. For extremely complex shapes the operator can use the robot simulation package in order to generate the final robot 
program. Both options are available for the user to decide.

7. Several case studies were carried out to prove the feasibility of the whole process and also the reliability of the slicing routines. Components with different levels of complexity were created with satisfactory success.

8. To assess the final metallic components, some representative mechanical tests were carried out. The tests performed were hardness tests, tensile tests, mechanical tests and micro examination. These prove that the components created with this process are metallurgically acceptable although all these components should undergo an in-depth study concerning quality and microstructural integrity.

\section{ACKNOWLEDGEMENTS}

First, I would like to thank my wife Marisa for encouraging and helping me on the difficult moments and my parents and sister for giving me strong support.

I would like to express my special gratitude to John Norrish for his wonderful supervision, guidance and interest in this work. For the academic help, I would like to thank Dr. R. S. McMaster. Special thanks go to John Savill for his strong support in the making of all the components and in solving in a very quick and efficient way all the technical problems. Thanks also go to the welding group for their strong support specially Babatunde Ogunbiyi for discussing and arguing with me all the time about our work.
[1] Antonio Fernando Ribeiro e John Norrish, 'Practical case of Rapid Prototyping using Gas Metal Arc Welding', Fifth International Conference on 'Computer Technology in Welding' - 15 a 16 de Junho de 1994, paper 55, Paris, França.

[2] Antonio Fernando Ribeiro e John Norrish, 'Rapid Prototyping for more complex shapes', $6^{\text {th }}$ Conferencia Internacional Bienal em 'Computer Technology in Welding' - 9-12 Junho de 1996, paper 60, Lanaken, Bélgica.

[3] Antonio Fernando Ribeiro e John Norrish, 'Rapid Prototyping Process using Metal Directly', Solid Freeform Fabrication 1996 Symposium - 12-14 Agosto de 1996, page 249, Austin, Texas, EUA.

[4] Antonio Fernando Ribeiro e Prof. Jonh Norrish, 'Case Study of Rapid Prototyping using Robot Welding - 'Square to Round' shape', $27^{\text {th }}$ International Symposium on Industrial Robotics, 6-8 October 1996, page 275, Milão, Itália.

[5] Antonio Fernando Ribeiro e John Norrish, 'Rapid Prototyping using Robot Welding - Process Concept and Experimental Arrangements', International Conference on Manufacturing Automation (ICMA'97), 28-30 de Abril de 1997, Hong Kong. 